\title{
Association of Peer Acceptance and Depressive Symptoms among High School Student in DKI Jakarta
}

\author{
Shitta Mutyahara, Wuri Prasetyawati ${ }^{1,2}$ \\ ${ }^{1,2}$ Department of Psychology, University of Indonesia. Indonesia \\ E-mail: wprasetyawati@gmail.com
}

\begin{abstract}
Psychological distress is known as one of the most common mental health problems among adolescents which are manifested in depression and anxiety symptoms. There are several factors that contribute to the emerging symptoms of mental health problems. For adolescents, one of the most important contributing factors is peer relationship as adolescents are getting more involved with their peers and less with their families. The aim of this study is to investigate the correlation between peer acceptance and depressive symptoms among high school student in DKI Jakarta. Perceived Acceptance Scale (PAS) and Hopkins Symptoms Check-List 25 (HSCL-25) were used to measure peer acceptance and depressive symptoms. Peer acceptance was hypothesized to be associated with depressive symptoms. The study was conducted on 767 high school students in 10th grade from DKI Jakarta. The result of this study showed a significant negative correlation between peer acceptance and depressive symptoms $(\mathrm{r}=-0.113, \mathrm{p}=0.002)$. Further research should examine other factors that influenced peer acceptance and depressive symptoms, along with the dynamics of peer relationship.
\end{abstract}

Keywords: adolescent; depressive symptoms; mental health; peer acceptance; psychological distress

\section{Introduction}

Adolescence is a crucial transition phase between childhood and adulthood. In this phase, there are physical, cognitive, emotional, and psychosocial changes in many different contexts such as social, culture, and economics (Papalia \& Martorell, 2013). Because of those changes, adolescents face new social responsibility; if they have any difficulties while adapting and solving the problem in their life, the stress level could increase, and the prevalence of mental health problems would also increase (Chandra \& Batada, 2006).

Adolescence is the phase when mental health problems are detected for the first time (Patel, Flisher, Hetrick, \& McGorry, 2007). The prevalence of adolescents having mental health problems is higher than children (Papalia \& Martorell, 2013). The study shows that one out of four adolescents in general population is having a mental health problem, ranging from age 12-24 years old (Patel et al., 2007). In Indonesia, the national survey (Riskesdas, 2013) shows that the prevalence of Indonesian with age 15-24 years old having mental health problems is $5.6 \%$ or at least 2.3 million adolescents in Indonesia is having mental health problems.

One of the most common mental health problems that occur in adolescence is psychological distress that is manifested in anxiety and depressive symptoms. Data from World Health Organization (n.d.) shows that depression ranks first for the cause of years lost to disability (YLD) for the age 15-19 years old. This means depression can inhibit adolescents' productivity. Furthermore, National Institute of Mental Health (2017) said that depression is common among adolescents, and the 
prevalence of adolescence is reaching $20 \%$ (Zuckerbrot \& Jensen, 2006).

Depression tends to be associated with sadness and hopelessness. However, depression in adolescence not only appears as sadness, the symptoms might appear as irritability, feeling bored, or the inability to feel happiness (Papalia \& Martorell, 2013). An article by WebMD.com (2017) points out a few signs of depression in adolescence like difficulty to concentrate and making decision, emergence of psychosomatics' complaint, change in eating and sleeping pattern, doing irresponsible acts (cutting off class, skip school, forgetting responsibility, and many more), thinking about death and suicide, bad grades, drinking alcohol and using drugs, withdrawing from social interactions, and many more. In this study, the depressive symptoms that we measure are the withdrawal of life interest, lack of motivation, and loss of vital energy (Derogatis et al., 1974). If these symptoms keep happening and adolescent do not get the right help, these depressive symptoms can develop into clinical depression.

Adolescents' daily life in an urban city like Jakarta lives with full of pressure and stressor. They become more susceptible to stress compared to the adolescents who live in the rural and village area (Romans, Cohen, \& Forte, 2011). High rates of criminality, conflict with friends and family, the compact daily routine, higher competitiveness, and worries about one academic achievement could influence the level of stress for adolescents who live in the urban city (Allison et al., Dise-Lewis et al., in Vera et al., 2012).

Support is needed from various parties to help adolescents with their problems. One of the sources of support which is closest to the adolescents is their peer. Adolescents spend more time with their peer compared to when they were in their childhood (Papalia \& Martorell, 2013). Peer is important for adolescent because peer becomes a source of affection, sympathy, care, and moral guidance, also as a place to do some experiment in social relationship and place to get autonomy, also independency from their parents (Papalia \& Martorell, 2013). Having a relationship with peer also helps adolescent to develop their interpersonal skill both for friendship and romantic relationship (Connoly, Furman, \& Konarski, 2000; Furman \& Wehner, 1994; in McElhaney, Antonishak, \& Allen, 2008).
Peer acceptance is defined as the relationship between the individual and member of their peer group, involves a sentiment (like or dislike), and that sentiment is supported by the other members of the peer group (Ladd, Kochenderfer, \& Coleman, 1997). Adolescents accepted by their peer will develop a sense of belonging in the peer group (Parker \& Asher, 1993) whereas a sense of belonging is important for adolescent's social and mental development (Sentse, Lindenberg, Omvlee, Ormel, \& Veenstra, 2010). In contrast, adolescents who are not well accepted or maybe rejected have a risk of having emotional problems and behavioral maladjustment (Kupersmidt \& Coie, 1990; Parker \& Asher, 1987; Rubin et al., 1998 in Sentse et al., 2010). A study in Italy shows that low peer status predicts self-reported depressive symptoms (Kiesner, 2002). There are a few depression risk factors for adolescents correlated with an interpersonal relationship such as low peer acceptance, loneliness, and self-reported victimization (Epkins \& Heckler, 2011). Another study showed that peer acceptance is one of the protective factors for internalizing symptoms; one of them being depression (Steinhausen \& Metzke, 2001).

This study aimed to see the correlation between peer acceptance and depressive symptoms among adolescents in Jakarta, Indonesia. The research hypothesized that there is an association between peer acceptance and depressive symptoms.

\section{Methods}

Sample. Participants of this study were 767 high school students in 10th grade from five high schools represented for five urban cities in Jakarta province, ranging between 14 to 22 years old. The sample was $52,7 \%$ female and $84,3 \%$ in the range of age 15 to 16 years old. Those five schools were selected by multistage random sampling with several inclusion and exclusion criterion. First, the researcher obtained the list of high school that was under the Ministry of Education and Culture in each city. Then researcher randomized the school and chose one for every city. Thus there were five schools that were chosen. All the 10th grader in the school then became the participant of this study.

Research Design. This was a one-shot school-based quantitative study. The descriptive analysis technique was used to compute the demographic data, and the Pearson Product Moment analysis technique was used to see the relationship between the two variables. 
Instrument and Measurement. Peer Acceptance. Participants completed Indonesian version of Perceived Acceptance Scale (PAS; Brock et al., 1998), the Friends subscale. The Friends subscale was translated into Indonesian using expert judgment and back-translation procedure, involving two experienced researchers who translated the questionnaire. Reliability procedures were used to ensure that the instruments were adequate to be used in this study. Cronbach's alpha for the Friends subscale was 0.7 . Validity analysis procedure by correlating PAS Friend with Social Support Questionnaire (SSQN) as a convergent and discriminant construct validity check has also been done by Brock et al (1998). The result of validity analysis was $r=-0.36$ for PAS Friend and perceived support by a friend. The correlation was significant at 0.001 levels.

Depressive Symptoms. Participants completed Indonesian version of Hopkins Symptoms Checklist 25 (HSCL-25; Derogatis et al., 1974). The Indonesian version of the instrument already translated and used by experts (Turnip \& Hauff, 2007). The reliability of the instrument was 0.928 (Patria, 2012).

Procedure. Recruitment of participants was conducted with multistage random sampling technique. All 10th grader in the selected school is chosen as participants. The inform consent was sent to the school and signed by the headmaster, described the project, the voluntary nature of participation, and the confidentiality of the data collected. The five chosen school agreed to participate in this study. The questionnaires were administered to the participants in schoolrooms during the regular class time at the convenience of the teachers. The questionnaires were administered individually by the researcher, and the participant answers to questionnaires independently.

\section{Results}

Most of the participants were 15-16 years old; most of them are female, and most of them going to school in West Jakarta. The mean of depressive symptoms score on the HSCL-25 in the current sample is $\mathrm{M}=2.44, \mathrm{SD}=0.666$ ), meanwhile the mean of peer acceptance score on the PAS Friends is $\mathrm{M}=37.32, \mathrm{SD}=5.08$.
Table 1

Correlation between Peer Acceptance and Depressive Symptoms

\begin{tabular}{cccc}
\hline \hline Variable & $\mathbf{R}$ & $\mathbf{p}$ & $\mathbf{N}$ \\
\hline Peer & & & \\
Acceptance & & .002 & 767 \\
Depressive & -.113 & & \\
symptoms & & & \\
\hline \hline
\end{tabular}

Table 1 shows the result of this study, there was a significant negative correlation between peer acceptance and depressive symptoms $(\mathrm{r}=-0.113, \mathrm{p}=$ 0.002). It means if the peer show higher acceptance for the adolescent, the adolescent tend to show lower depressive symptoms, vice versa.

\section{Discussion \& Conclusion}

Discussion. The result of the correlational analyses showed that there is a significant negative correlation between peer acceptance and depressive symptoms in adolescents. This pattern of findings is similar to the study conducted by Steinhausen and Metzke (2001), which showed peer acceptance as one of the protective factors of internalizing symptoms, one of them being depression. Another study by Sentse et al. (2010) is also consistent with the finding; that low peer acceptance is correlated to internalizing problems; one of them is depressive symptoms. Peer acceptance becomes a protective factor while peer rejection is associated with higher level of internalizing problems, and peer acceptance is associated with lower level of internalizing problems. This shows that adolescents wellaccepted in their peer tend to show lower depressive symptoms, and vice versa.

The questionnaire responses for depressive symptoms showed that $82.7 \%$ of participants of this study have a high level of depressive symptoms. This might be caused by the developmental characteristics of adolescent, as they go through physical, cognitive, and psychosocial changes in their current phase of life. Changes in a relationship with peers, new academic pressure and challenge, conflict with parents, and other life events make them more vulnerable to stress, and that might develop to emotional problems, which then lead to psychological distress (Center for Adolescent Health, n.d.). Besides said factors, urban city lifestyle can be another stressor for adolescents. High criminality rate around in urban cities and 
exposure to crime, conflict with family and friends, tight schedule of their daily routine, higher level of competitiveness, and the worries of their academic achievement could influence the level of stress of adolescent who live in the city (Allison et al., DiseLewis et al., in Vera et al., 2012), such as DKI Jakarta. Heavy traffic of the city, pollution, and the distance between their home and school could also be another stressor for adolescents.

There are some limitations to the design of this study. First, the researcher did not do any reliability and validity test to the Indonesian version of HSCL25 in the sample because the instrument had already been tested before and has been shown to have good reliability and validity score. Second, the researcher used 1.75 as a cut-off point to determine the level of depressive symptoms based on the previous study. As the prevalence of depressive symptoms in this sample is quite high, there is a chance that 1.75 as cut-off point is not suitable for the sample that lives in the city.

Conclusions. From this study, we found that there is a significant negative correlation between peer acceptance and depressive symptoms. When adolescent perceived that they are well accepted by their peer, they tend to show lower depressive symptoms. Meanwhile, when adolescent perceived that they are not accepted by their peer, they tend to show higher depressive symptoms.

This finding can be used as an additional literature on the topics of peer relationship and depressive symptoms. This finding can also help the school counselor or psychologist to make a preventive program for their students about peer relationship and depression and the link in between. This finding may also contribute to the previous research (Steinhausen \& Metzke, 2001; Sentse et al., 2010) in a different cultural context. Future research could involve qualitative methods such as interview to explore the stressors for the adolescents and what they feel when facing it on a daily basis. Also exploring other factors that influence peer acceptance and depressive symptoms, along with the dynamics of peer relationship.

\section{References}

Brock, D., Sarason, I., Sanghvi, H., \& Gurung, R. (1998). The Perceived Acceptance Scale: Development and validation. Journal Of Social And Personal Relationships, $\quad 15(1)$ http://dx.doi.org/10.1177/0265407598151001
Chandra, A., \& Batada, A. (2006). Exploring stress and coping among urban African American adolescents: the Shifting the Lens study. Preventing Chronic Disease, 3

Derogatis, L., Lipman, R., Rickels, K., Uhlenhuth, E., \& Covi, L. (1974). The Hopkins Symptom Checklist (HSCL): A self-report symptom inventory. Behavioral Science, 19(1), 1-15. http://dx.doi.org/10.1002/bs.3830190102

Epkins, C., \& Heckler, D. (2011). Integrating etiological models of social anxiety and depression in youth: Evidence for a cumulative interpersonal risk model. Clinical Child And Family Psychology Review, 14(4), 329-376. http://dx.doi.org/10.1007/s10567-011-0101-8

Kiesner, J. (2002). Depressive symptoms in early adolescence: Their relations with classroom problem behavior and peer status. Journal Of Research On Adolescence, 12(4), 463-478. http://dx.doi.org/10.1111/1532-7795.00042

Ladd, G., Kochenderfer, B., \& Coleman, C. (1997). Classroom peer acceptance, friendship, and victimization: Distinct relational systems that contribute uniquely to children's school adjustment?. Child Development, 68(6). http://dx.doi.org/10.2307/1132300

NIMH » Major Depression Among Adolescents. (2017). nimh.nih.gov. Retrieved from https://www.nimh.nih.gov/health/statistics/prevalence /major-depression-among-adolescents.shtml.

McElhaney, K., Antonishak, J., \& Allen, J. (2008). "They Like Me, They Like Me Not": Popularity and adolescents' perceptions of acceptance predicting social functioning over time. Child Development, 79(3), 720-731. http://dx.doi.org/10.1111/j.1467-8624.2008.01153.x

Papalia, D. E., \& Martorell, G. (2013). Experience human development (13th ed.). New York: McGraw-Hill Education.

Parker, J., \& Asher, S. (1993). Friendship and friendship quality in middle childhood: Links with peer group acceptance and feelings of loneliness and social dissatisfaction. Developmental Psychology, 29(4), 611-621. http://dx.doi.org/10.1037//0012-1649.29.4.611

Patel, V., Flisher, A., Hetrick, S., \& McGorry, P. (2007). Mental health of young people: a global public-health challenge. The Lancet, 369(9569), 1302-1313. http://dx.doi.org/10.1016/s0140-6736(07)60368-7

Patria, F. Y. (2012). The relationship between sense of community and psychological distress among members of Faculty of Psychology Universitas Indonesia. Undergraduate thesis. Universitas Indonesia: Faculty of Psychology. Hubungan Antara sense of community dengan distress psikologik pada Warga Fakultas Psikologi Universitas Indonesia. Skripsi. Universitas Indonesia: Fakultas Psikologi.

Romans, S., Cohen, M., \& Forte, T. (2011). Rates of depression and anxiety in urban and rural Canada. Social Psychiatry and Psychiatric Epidemiology, 46, 567-575.

Sentse, M., Lindenberg, S., Omvlee, A., Ormel, J., \& Veenstra, R. (2010). Rejection and acceptance across contexts: Parents and peers as risks and buffers for early 
adolescent psychopathology. The TRAILS Study. Journal Of Abnormal Child Psychology, 38(1), 119-130. http://dx.doi.org/10.1007/s10802-009-9351-z

Steinhausen, H., Metzke, C. (2001). Risk, compensatory, vulnerability, and protective factors influencing mental health in adolescence. (2001). Journal Of Youth And Adolescence, 30(3).

Teen Depression: Symptoms and Tips for Parents. (2017). WebMD. Retrieved

from http://www.webmd.com/parenting/teen-abuse-coughmedicine-9/depression

Teen stress. (n.d.). Center for Adolescent Health. Retrieved from http://www.jhsph.edu/research/centers-andinstitutes/center-for-adolescent-health/_includes/_preredesign/Teen_Stress_Standalone.pdf
Vera, E. M., Vacek, K., Blackmon, S., Coyle, L., Gomez, K., Jorgenson, K., . . Steele, J. C. (2012). Subjective wellbeing in urban, ethnically diverse adolescents: The role of stress and coping. Youth and Society, 44(3), 331-347.

World Health Organization. (n.d.). Adolescent health epidemiology. Retrieved from http://www.who.int/maternal_child_adolescent/epide miology/adolescence/en/

Zuckerbrot, R., \& Jensen, P. (2006). Improving recognition of adolescent depression in primary care. Archives of Pediatrics \& Adolescent Medicine, 160(7), 694. http://dx.doi.org/10.1001/archpedi.160.7.694 